\title{
LAS MEDIDAS EXTRAORDINARIAS ADOPTADAS EN ESPAÑA Y EUROPA EN LOS SEGUROS Y EN LOS PLANES DE PENSIONES PARA COMBATIR LAS CONSECUENCIAS DE LA PANDEMIA DEL COVID-19
}

\section{EXTRAORDINARY MEASURES TAKEN IN SPAIN AND EUROPE IN INSURANCE AND PENSION PLANS TO COMBAT THE CONSEQUENCES OF THE COVID PANDEMIC 19}

\author{
ALBERTO J. TAPIA HERMIDA* \\ Fecha de recepción: 4 de junio 2020 \\ Fecha de aceptación 15 junio 2020 \\ Disponible en línea: 30 de junio 2020
}

Para citar este artículo/To cite this article

\begin{abstract}
TAPIA Hermida, Alberto J. Las medidas extraordinarias adoptadas en España y Europa en los seguros y en los planes de pensiones para combatir las consecuencias de la pandemia del COVID-19, 52 Rev.Ibero-Latinoam.Seguros, 15-36 (2020). https://doi.org/10.11144/Javeriana.ris52.meae
\end{abstract}

Doi: $10.11144 /$ Javeriana.ris52.meae

\footnotetext{
* Licenciado en Derecho por la Universidad Autónoma de Madrid. Doctor en Derecho por la Universidad Complutense de Madrid. Es Abogado de ESTUDIO JURÍDICO Sánchez Calero. Ha sido Letrado de la Comisión Nacional del Mercado de Valores. Es experto en Derecho de seguros y ha intervenido en numerosos litigios relativos a este sector. También tiene una dilatada experiencia en mercados financieros y en Derecho bancario y bursátil. Emite dictámenes e informes en asuntos mercantiles y ha sido requerido para actuar como experto en procedimientos judiciales y arbitrales. Catedrático Acreditado de Derecho mercantil. Facultad de Derecho. Universidad Complutense de Madrid. Contacto: atapia@sanchezcalero.com; ORCID: https://orcid.org/0000-0002-5736-1611
} 


\section{RESUMEN}

Este estudio examina las medidas extraordinarias adoptadas en España y Europa en los seguros y en los planes de pensiones para combatir las consecuencias de la pandemia del COVID-19 comenzando por destacar su utilidad para los países iberoamericanos. Su contenido se distribuye en cuatro apartados que se refieren a las medidas extraordinarias adoptadas en el estatuto de las entidades aseguradoras (remuneración de los administradores y del personal directivo e información por las personas y entidades sujetas a supervisión), en los seguros de daños (el refuerzo del seguro de crédito a la exportación en Europa y en España), en los seguros de personas (el seguro de vida y la Póliza de protección del personal sanitario que luchan contra el COVID-19) y en los planes de pensiones (disponibilidad de los derechos consolidados).

Palabras Clave: COVID-19, seguros, planes de pensiones, seguro de crédito a la exportación, seguro de vida, Póliza de protección del personal sanitario que luchan contra el COVID-19. 


\begin{abstract}
This study examines the extraordinary measures taken in Spain and Europe in insurance and pension plans to combat the consequences of the COVID pandemic 19 , beginning by highlighting their usefulness for Ibero-American countries. Its content is distributed in four sections that refer to the extraordinary measures adopted in the statute of insurance entities (remuneration of administrators and management personnel and information by persons and entities subject to supervision), in damage insurance (the strengthening of export credit insurance in Europe and Spain), in personal insurance (life insurance and the Protection policy for health personnel fighting against COVID-19) and in pension plans (availability of consolidated rights).
\end{abstract}

Keywords: COVID-19, insurance, pension plans, export credit insurance, life insurance, Protection policy for health personnel fighting against COVID-19.

\title{
SUMARIO
}

I. Presentación: sobre la utilidad de este estudio para los países iberoamericanos. II. Medidas extraordinarias en el estatuto de las entidades aseguradoras. A) Remuneración de los administradores y del personal de las entidades aseguradoras en tiempo del coronavirus. Directrices europeas. B) Información por las personas y entidades sujetas a la supervisión de la Dirección General de Seguros y Fondos de Pensiones. Normas españolas. III. Medidas extraordinarias en los seguros de daños. A) La Comunicación de la Comisión Europea de 28 de marzo de 2020 para adaptar el seguro de crédito a la exportación a corto plazo a las circunstancias extraordinarias provocadas por el brote de COVID-19. B) El refuerzo en España del Seguro de Crédito a la Exportación como medida extraordinaria de apoyo público a la financiación empresarial ante la crisis del COVID-19 por el Real Decreto-Ley No 8/2020. IV. Medidas y experiencias extraordinarias en los seguros de personas. A) El seguro de vida en época del COVID-19. 1. Seguros de vida vigentes. 2. Seguros de vida nuevos. 3. La aplicación del método de diagnóstico precoz de las 5 preguntas a la situación pandémica global del COVID-19. B) La póliza de protección de los sanitarios que luchan contra el COVID-19. Una iniciativa admirable de las aseguradoras españolas a través de UNESPA. 1. Un colectivo admirable e incluso heroico: los profesionales sanitarios en lucha frente al COVID-19. 2. Una póliza de seguro de vida admirable. 3. Una póliza de arquitectura original. 3.1. Elementos personales de la Póliza. 3.2. Contenido de la Póliza. 4. Una demostración de responsabilidad social corporativa. 5. Conclusión: una Póliza de Seguro de Vida histórica. V. Medidas extraordinarias en los planes de pensiones. A) El marco inicial de la disponibilidad de los planes de pensiones por la crisis del COVID-19 establecido en el RDL 11/2020. B) El desarrollo de las normas sobre la disponibilidad de los planes de pensiones por la crisis del COVID-19 por el RDL 15/2020. C) Reformas de la disponibilidad de planes de pensiones por el RDL 16/2020. VI. Conclusiones. Bibliografía. 


\section{PRESENTACIÓN: SOBRE LA UTILIDAD DE ESTE ESTUDIO PARA LOS PAÍSES IBEROAMERICANOS}

España ha sido, por desgracia, uno de los países pioneros en sufrir la pandemia del COVID-19 que ha causado entre nuestra población más de 40.000 muertes. Es por ello por lo que esta dramática experiencia puede servir para sacar, en el mundo del seguro, enseñanzas que puedan resultar útiles para los países hermanos de Iberoamérica que se dirigen a sufrir, en los próximos meses, las consecuencias de estar en el epicentro de la expansión del COVID-19.

Como ejemplo de lo anterior, podemos referirnos a la relevancia de los seguros de personas en época del COVID-19. Así vemos como, en esta época en la que vivimos sumidos en la tragedia humana, social, política y económica ocasionada por la pandemia provocada por el COVID-19; cuando las incertidumbres y los consiguientes riesgos de toda especie sobre lo que nos deparará el futuro próximo y lejano atenazan nuestras conciencias y voluntades; el seguro, como instrumento que permite la cobertura recíproca de necesidades eventuales - por ser futuras e inciertas- a un costo parcial se nos presenta como un mecanismo socialmente deseable por útil ${ }^{1}$.

En particular, la crisis causada por la pandemia global del COVID-19 ha mostrado en España dos características relevantes a la hora de proyectar la cobertura aseguradora de nuestras vidas y haciendas que son:

a) Por un lado, la certeza de que esta crisis sanitaria se prolongará, cuando menos, hasta el año 2022, conforme a un estudio reciente de científicos de la muy prestigiosa Universidad de Harvard. Mientras que la crisis económica se extenderá, como mínimo, hasta el año 2024, conforme a previsiones prudentes e informadas. Duración que nos lleva a calificar de época el periodo de tiempo infausto que nos ha tocado vivir.

b) Por otro lado, la incertidumbre sanitaria específica generada por la escasez de test de todo tipo que permitan descartar o confirmar la presencia del COVID-19 y de los equipos elementales de prevención y protección frente al virus (EPIs, mascarillas, etc.).

Este incertidumbre se potencia, con consecuencias relevantes con vistas a la prevención de riesgos futuros, porque la especial conformación del COVID-19 conduce a la identificación de 4 grupos básicos de población desde un punto de vista epidemiológico que son: Los sujetos no infectados, los infectados asintomáticos, quienes muestran síntomas leves y aquellos en los que la enfermedad cursa con síntomas graves (fundamentalmente, neumonías bilaterales) que, a su vez, podrían clasificarse en dos subgrupos, conforme requieran hospitalización y esta se les depare o no (personas de avanzada edad en residencias de ancianos, etc.). Ello genera una incertidumbre añadida que multiplica geométricamente los riesgos sanitarios, su prevención y su eventual cobertura mediante el seguro.

\footnotetext{
1 V. la entrada de nuestro blog (ajtapia.com) de 20.04.2020 sobre "El seguro de vida en época del COVID-19”.
} 


\section{MEDIDAS EXTRAORDINARIAS EN EL ESTATUTO DE LAS ENTIDADES ASEGURADORAS}

\section{A) Remuneración de los administradores y del personal de las entidades aseguradoras en tiempo del coronavirus. Di- rectrices europeas}

La Autoridad Europea de Seguros y Pensiones de Jubilación (conocida por sus acrónimos en inglés como EIOPA) ${ }^{2}$ publicó, el día 2 de abril de 2020, una "Declaración sobre distribución de dividendos y remuneración variable. Politicas en el contexto del COVID-19”3. que siguió la dirección del previamente publicado el 17 de marzo. Adviértase que los principios armonizados de supervisión de las remuneraciones que se establecen en la Opinión de 31 de enero siguen siendo válidos en la actual situación excepcional originada por la crisis brutal y misteriosa del COVID-19, matizados por los criterios específicamente fijados por EIOPA a los que no referimos seguidamente; $\mathrm{y}$, sobre todo, seguirán siendo válidos cuando esta pandemia infausta nos diga adiós.

En esta Declaración EIOPA reitera lo ya dicho en su Declaración del pasado 17 de marzo, en el contexto de la crisis actual del COVID-19 en el sentido siguiente: "Todas las aseguradoras y reaseguradoras deben tomar medidas para preservar su posición de capital en equilibrio con la protección del asegurado, repartiendo un dividendo prudente y otras políticas de distribución, incluida la remuneración variable". Dicha prudencia se proyecta en que.

"las aseguradoras y reaseguradoras deben asegurarse de que la evaluación de sus necesidades generales de solvencia son prospectivas, teniendo debidamente en cuenta

\footnotetext{
2 EIOPA es una de las Autoridades que integran el Sistema Europeo de Supervisión Financiera (SESF/ ESFS). Este SESF nació en el año 2011 como una solución al problema de la descoordinación supervisora detectada en el seno de la UE con ocasión de la crisis financiera de 2008. Por ello, la implantación del SESF tuvo como propósito general recobrar la simetría entre el sujeto supervisor y el objeto supervisado subsanando los graves problemas de descoordinación de las autoridades nacionales encargadas de la supervisión financiera que se manifestaron en la Unión Europea. De tal manera que el SESF se crea como una red integrada de las autoridades supervisoras nacionales y comunitarias. El SESF se articuló jurídicamente mediante la promulgación de cuatro Reglamentos del Parlamento Europeo y del Consejo (los n ${ }^{\circ} 1092$, 1093, 1094 y 1095, de 2010), de un Reglamento del Consejo (el n 1096, de 2010) y de una Directiva del Parlamento Europeo y del Consejo (2010/78/UE) que modifica otras precedentes. Ver nuestro estudio sobre "El Sistema Europeo de Supervisión Financiera", en la Revista de Derecho Bancario y Bursátil núm.121 de 2011; así como le entrada de nuestro blog ajtapia.com) del 3 octubre de 2017 sobre La Comisión Europea propone reforzar el Sistema Europeo de Supervisión Financiera y las que en ella se mencionan. En nuestro blog (ajtapia.com) nos hemos ocupado en numerosas ocasiones de EIOPA y la labor que desarrolla. En este sentido, se pueden ver las entradas de 30 mayo de 2017 sobre Seguros de vida unit-linked. Máxima transparencia en su gestión y en su distribución. Documento de EIOPA sobre los productos de inversión basados en seguros y de 16 octubre de 2015 sobre La Autoridad Europea de Seguros y Pensiones de Jubilación (EIOPA) publica nuevas Directrices para la implantación de Solvencia II y las que en ellas se mencionan. 3 Statement on dividends distribution and variable remuneration policies in the context of COVID-19. Ver las entradas de nuestro blog (ajtapia.com) de 13 y 14 de abril de 2020 tituladas "Aseguradoras. Remuneración de sus administradores y de su personal en tiempo del coronavirus. Declaración de EIOPA de 2 de abril de 2020”.
} 
el actual nivel de incertidumbre sobre la profundidad, magnitud y duración de los impactos del COVID-19 en los mercados financieros y en la economía y las repercusiones de esta incertidumbre en su solvencia y posición financiera"4.

Por lo anterior, EIOPA acaba instando a que las entidades aseguradoras y reaseguradoras, en el contexto de incertidumbre causado por la crisis actual del COVID-19: "Suspendan temporalmente todas las distribuciones de dividendos discrecionales y contemplen recompras de acciones destinadas a remunerar a los accionistas. Esta suspensión debe ser revisada a medida que el impacto financiero y económico del COVID-19 comience a determinarse con mayor claridad".

\section{B) Información por las personas y entidades sujetas a la su- pervisión de la Dirección General de Seguros y Fondos de Pensiones. Normas españolas}

El Art. 24 del Real Decreto-Ley $N^{\circ}$ 15/2020, de 21 de abril, de medidas urgentes

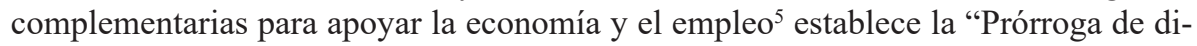
versos términos y plazos de presentación de información por las personas y entidades sujetas a la supervisión de la Dirección General de Seguros y Fondos de Pensiones" que afecta a las entidades siguientes.

a) En el ámbito de la ordenación y supervisión de las entidades aseguradoras y reaseguradoras: Los plazos relativos a la presentación del Informe Periódico de Supervisión durante el año 2020; la presentación ante la autoridad supervisora y la publicación del Informe de Situación Financiera y de Solvencia referida al cierre del ejercicio económico 2019 y del correspondiente Informe Especial de Revisión; y la presentación ante la autoridad supervisora de la información cuantitativa, o estadístico-contable, anual por el ejercicio económico 2019 y trimestral por el primer trimestre del ejercicio económico 2020.

b) En el ámbito de la ordenación y supervisión de los planes y fondos de pensiones, así como de las entidades gestoras y depositarias de estos fondos: Los plazos y términos relativos a la presentación ante la autoridad supervisora del Informe sobre el grado de cumplimiento de las normas de separación entre la entidad gestora y la depositaria; del Informe sobre la efectividad de los procedimientos de control interno de las entidades gestoras de fondos de pensiones; de la Revisión financiero actuarial a la que se refiere el Art. 23 del Reglamento de planes y fondos de pensiones, aprobado por el Real Decreto 304/2004, de 20 de febrero; y de la información estadística, financiera y contable, a efectos de supervisión, de los fondos de pensiones que actúan en España y de sus entidades gestoras, correspondiente al cierre del ejercicio económico 2019 y al primer trimestre del ejercicio económico 2020.

c) En el ámbito de la ordenación y supervisión de los distribuidores de seguros y reaseguros: Los plazos y términos relativos a la presentación ante la autoridad super-

\footnotetext{
${ }^{4}$ La traducción del documento original en inglés es nuestra.

5 BOE núm. 112 del miércoles 22 de abril de 2020.
} 
visora de la información estadístico-contable y de negocio correspondiente al cierre del ejercicio económico 2019.

\section{MEDIDAS EXTRAORDINARIAS EN LOS SEGUROS DE DAÑOS}

\section{A) La Comunicación de la Comisión Europea de 28 de marzo de 2020 para adaptar el seguro de crédito a la exportación a corto plazo a las circunstancias extraordinarias provocadas por el brote de COVID-19}

La Comunicación de la Comisión Europea de 20 de marzo de 2020 sobre las ayudas estatales por el COVID en la Unión Europea identifica 5 tipos de medidas temporales de ayuda estatal y las respectivas condiciones que deben cumplir para que la Comisión Europea considere que tales ayudas estatales resultan compatibles con el mercado interior con arreglo al Art. 107, apartado 3, letra b), del TFUE6 ${ }^{6}$. La última de ellas se refiere al "seguro de crédito a la exportación a corto plazo".

Después, la Comunicación de la Comisión por la que se modifica el anexo de la Comunicación de la Comisión a los Estados miembros sobre la aplicación de los Arts. 107 y 108 del Tratado de Funcionamiento de la Unión Europea al seguro de crédito a la exportación a corto plazo $^{7}$ tiene por finalidad adaptar la Comunicación de la Comisión de 2012 a los Estados miembros sobre la aplicación de los Arts. 107 y 108 del Tratado de Funcionamiento de la Unión Europea al seguro de crédito a la exportación a corto plazo $^{8}$ a las circunstancias extraordinarias provocadas por el brote de COVID-19. Y ello porque el repentino brote de COVID-19 en los primeros meses de 2020 ha supuesto uno solo una seria amenaza para la salud pública a escala mundial, sino que ha provocado, además, grandes perturbaciones en la economía mundial y en la economía de la Unión. En particular, como consecuencia de este brote, las empresas se enfrentan a graves problemas de liquidez y su situación comercial ha quedado expuesta a mayores riesgos financieros.

Ante esta situación, la Comisión ha basado su evaluación de la situación en los criterios establecidos en el punto 33 de la Comunicación de 2012 (capacidad privada de seguro de crédito, calificación soberana y rendimiento del sector empresarial) y el 23 de marzo de 2020 publicó una solicitud de información sobre la disponibilidad de seguros de exportación a corto plazo para las exportaciones a todos los países cuyos riesgos se consideran negociables con arreglo a la Comunicación. De la consulta ha resultado que la economía mundial y la economía de la Unión están experimentando una contracción debida al brote de COVID-19, según los indicadores macroeconó-

\footnotetext{
${ }^{6}$ V. la entrada de nuestro blog (ajtapia.com) del pasado 25 de marzo sobre "Las ayudas estatales por el coronavirus en la Unión Europea: la Comunicación de la Comisión Europea de 20 de marzo de 2020".

7 Ref. 2020/C 101 I/01; DOUE de 28.3.2020. Ver la entrada de nuestro blog (ajtapia.com) de 30.03.2020 sobre "El seguro de crédito a la exportación ante la crisis del COVID-19: Comunicación de la Comisión Europea de 28 de marzo de 2020”.

8 DOUE C 392 de 19.12.2012.
} 
micos más recientes y cabe esperar que a corto plazo se produzca un deterioro de las calificaciones soberanas como resultado de la crisis provocada por el brote de COVID-19. Se prevé que el rendimiento del sector empresarial se deteriore, dando lugar a una importante corrección de las previsiones de la Comisión, con un crecimiento negativo, en relación con las previsiones de invierno para la UE en 2020 (1,4 \%).

Dadas estas circunstancias, la Comisión ha decidido considerar todos los riesgos comerciales y políticos asociados a las exportaciones a los países enumerados en el anexo de la Comunicación como «temporalmente no negociables» hasta el 31 de diciembre de 2020, en consonancia con la duración del Marco Temporal ${ }^{9}$.

\section{B) El refuerzo en España del Seguro de Crédito a la Expor- tación como medida extraordinaria de apoyo público a la financiación empresarial ante la crisis del COVID-19 por el Real Decreto-Ley $\mathbf{N}^{\circ} 8 / 2020$}

El capítulo III del Real Decreto-Ley N 8/2020, de 17 de marzo, de medidas urgentes extraordinarias para hacer frente al impacto económico y social del COVID-19 (RDL $8 / 2020)^{10}$ establece diversas medidas de garantía de liquidez para sostener la actividad económica ante las dificultades transitorias consecuencia de la situación generada por el COVID-19. Entre estas medidas extraordinarias, para garantizar la liquidez de las empresas exportadoras, el RDL 8/2020 refuerza la capacidad de la Compañía Española de Seguros de Crédito a la Exportación, S.A. Cía. de Seguros y Reaseguros (CESCE) para el aumento de la cobertura por cuenta del Estado de sus garantías. Esta "línea extraordinaria de cobertura aseguradora" se desarrolla en el Art. 31 del RDL 8/2020 que, con carácter extraordinario y con una duración de 6 meses desde la entrada en vigor del RDL 8/2020, autoriza la creación de una línea de cobertura aseguradora de hasta 2.000 millones de euros con cargo al Fondo de Reserva de los Riesgos de la Internacionalización, con unas características determinadas referidas a los créditos elegibles de circulante necesarios para la compañía exportadora, a las empresas beneficiarias ${ }^{11}$.

\footnotetext{
9 En consecuencia, "a partir del 27 de marzo de 2020 y hasta el final de 2020 se aplicará la siguiente modificación de la Comunicación de la Comisión a los Estados miembros sobre la aplicación de los Arts. 107 y 108 del Tratado de Funcionamiento de la Unión Europea al seguro de crédito a la exportación a corto plazo: El anexo se sustituye por el texto siguiente: «Lista de países cuyos riesgos son negociables La Comisión considera temporalmente no negociables todos los riesgos comerciales y políticos asociados a las exportaciones a los países enumerados a continuación hasta el 31 de diciembre de 2020. Bélgica Chipre Eslovaquia Bulgaria Letonia Finlandia Chequia Lituania Suecia Dinamarca Luxemburgo Reino Unido Alemania Hungría Australia Estonia Malta Canadá Irlanda Países Bajos Islandia Grecia Austria Japón España Polonia Nueva Zelanda Francia Portugal Noruega Croacia Rumanía Suiza Italia Eslovenia Estados Unidos de América".

10 BOE núm. 73 del miércoles 18 de marzo de 2020. V. la entrada de nuestro blog (ajtapia.com) del pasado 19 de marzo sobre "Aspectos financieros y mercantiles de las medidas urgentes extraordinarias del Real Decreto-Ley N N $^{\circ} / 2020$ por el coronavirus (2)" y del 30 de marzo de 2020 sobre "El refuerzo del Seguro de Crédito a la Exportación como medida extraordinaria de apoyo público a la financiación empresarial ante la crisis del COVID-19 por el Real Decreto-Ley N 8/2020"

11 Son las empresas españolas consideradas como PYMES conforme a la definición del Anexo I del Reglamento UE 651/2014 de la Comisión y otras empresas de mayor tamaño, siempre que sean entidades no
} 
Más tarde, el RDL 15/2020 ha potenciado el seguro de crédito y de caución habilitando al Consorcio de Compensación de Seguros para que desarrolle actividades de reaseguro de crédito y de caución a partir de 2020. Para ello, el objetivo mencionado, el Art. 7 del RDL 15/2020 establece las "condiciones básicas de la aceptación en reaseguro por parte del Consorcio de Compensación de Seguros de los riesgos del seguro de crédito asumidos por las entidades aseguradoras privadas". Estas condiciones básicas del reaseguro aceptado por el Consorcio se refieren a los aspectos siguientes:

a) Modalidades de la cobertura, que deberán incluirse entre las comunes en el mercado de reaseguro, que permitan complementar con rapidez y eficacia la cobertura directa que las entidades aseguradoras de estos ramos ofrecen a las empresas por ellas aseguradas, contribuyendo a dar seguridad a las transacciones económicas.

b) Condiciones económicas que establecerá el con el objetivo de procurar el equilibrio financiero del acuerdo a largo plazo, contemplándose, dentro de las citadas condiciones, la compensación que corresponda por los gastos de gestión en que incurra el propio Consorcio.

c) Objeto y vigencia temporal ya que la cobertura podrá aplicarse, a partir del día 1 de enero de 2020, a las operaciones de seguro, que sean llevadas a cabo por entidades aseguradoras autorizadas en el ramo de crédito con un volumen de operaciones significativo, y cuyos asegurados estén domiciliados en España. Y su vigencia temporal se mantendrá en tanto subsistan las razones de interés general que justificaron su adopción y por un periodo mínimo de dos años.

Por otro lado, se regulan las condiciones actuariales y financieras en las que el Consorcio de Compensación de Seguros llevará las operaciones de aceptación del reaseguro que realice al amparo del RDL 15/2020.

\section{MEDIDAS Y EXPERIENCIAS EXTRAORDINARIAS EN LOS SEGUROS DE PERSONAS}

\section{A) E1 seguro de vida en época del COVID-19}

Dentro del panorama trágico que ha generado la crisis del COVID-19, nos parece que puede ser útil plantear -siquiera en dos pinceladas- los problemas derivados de los contratos vigentes y futuros de personas en general $\mathrm{y}$, en particular, de los que plantean los seguros de vida ${ }^{12}$. Y aquí nos parece pertinente distinguir -a los efectos

cotizadas, en las que concurran determinadas circunstancias. Sobre el seguro de crédito en general, puede verse nuestra Guía del Contrato de Seguro, Ed, Thomson Reuters Aranzadi, Cizur Menor 2018, pp. 114 y ss.

12 Recordemos que el seguro sobre la vida es un tipo de seguro de personas, regulado en la Sección 2. ${ }^{\text {a }}$ del Título III de la LCS (Arts. 83 a 89) por el que el asegurador se obliga, mediante el cobro de la prima estipulada y dentro de los límites establecidos en la Ley y en el contrato, a satisfacer al beneficiario un capital, una renta u otras prestaciones convenidas, en el caso de muerte o bien de supervivencia del asegurado, o de ambos eventos conjuntamente (el lector interesado en la materia puede consultar nuestra Guía del Contrato de Seguro, Ed. Reuters, Aranzadi, 1ª Edición, Cizur Menor 2018, pp. 155 y ss.). 
de su ubicación dentro de la pandemia provocada por el COVID-19-dos situaciones básicas que referimos seguidamente.

\section{Seguros de vida vigentes}

Respecto de estos seguros se plantea la evidente agravación del riesgo que implican la multitud de situaciones generadas por la pandemia global del COVID-19 y el juego del Art. 11 de la LCS que, en su apartado 2 dispone: "En los seguros de personas el tomador o el asegurado no tienen obligación de comunicar la variación de las circunstancias relativas al estado de salud del asegurado, que en ningún caso se considerarán agravación del riesgo". Por lo tanto, el tomador que se vea infectado por el COVID-19 no estará obligado a comunicarlo al asegurador como agravación del riesgo.

\section{Seguros de vida nuevos}

En aquellas situaciones en las que sea necesario o conveniente celebrar nuevos contratos de seguros de vida, el primero de los problemas se plantea en el momento en el que el tomador y asegurado deba cumplir con la obligación precontractual de declaración del riesgo mediante la respuesta al cuestionario de salud que le someta el asegurador, todo ello en los términos establecidos por el Art. 10 de la LCS.

Y decimos que la crisis causada por la pandemia global del COVID-19 provoca nuevos problemas porque, entonces, a los problemas propios de situaciones normales (presentación del cuestionario, contenido de sus preguntas, cumplimentación del mismo, etc.) se unirán los propios de esta pandemia $\mathrm{y}$, muy especialmente, los que derivarán, por un lado, de las incertidumbres nacidas de la carencia de test de todo tipo que permitan descartar o confirmar la presencia del COVID-19 y, por otro lado, de los diversos grupos básicos de población desde un punto de vista epidemiológico a los que antes nos referíamos (particularmente, los sujetos no infectados y los infectados asintomáticos).

A partir de ahora, habrá que tener en cuenta la situación pandémica global del COVID-19 a la hora de proyectar los criterios jurisprudenciales en la aplicación del Art. 10 de La LCS. Y así, recordar que, en términos generales, los tribunales tienen establecido que, para que se produzca la liberación del asegurador de su obligación de cumplimiento de la prestación ex Art. 10.3 LCS, tienen que concurrir una serie de presupuestos cumulativos: que haya presentado el cuestionario, que el contenido de éste sea claro, que pruebe que la ocultación de la enfermedad se debió a dolo civil o culpa grave del tomador y que entre la enfermedad ocultada y el siniestro se acredite una relación de causalidad eficiente [STS 23.11.2005 (RJ 2005, 7643)]. Por lo anterior, los tribunales han sostenido la cobertura íntegra por el asegurador en los casos en que concurra cualquiera de las circunstancias siguientes: cuando no se ha presentado por el asegurador el cuestionario de salud, cuando el cuestionario presentado sea poco claro, cuando aquel cuestionario se cumplimentó por un agente del asegurador 
sin intervención ni conocimiento del tomador o asegurado, cuando no se acredita la ocultación dolosa o gravemente culposa de la enfermedad o cuando falta la relación de causalidad eficiente entre la enfermedad preexistente no declarada y la enfermedad causante del siniestro [STS 15.12.2005 (JUR 2006, 19881)].

\section{La aplicación del método de diagnóstico precoz de las 5 preguntas a la situación pandémica global del COVID-19}

En relación con lo dicho, nos parece que puede ser extremadamente útil para los aseguradores, mediadores y asegurados aplicar a la situación pandémica global del COVID-19 el método de diagnóstico precoz de las 5 preguntas ${ }^{13}$. En el sentido anterior, el examen de la jurisprudencia reciente de la Sala Primera de lo Civil del Tribunal Supremo de España en materia de seguros en general y de seguros de vida en particular pone de manifiesto que uno de los tipos de litigios que llegan con mayor frecuencia al alto tribunal son los que versan sobre la eventual reducción o negación de la prestación del asegurador en los casos de omisión -por parte del tomador del seguro- de circunstancias relevantes para la valoración del riesgo en el cuestionario de salud de los seguros de vida, todo ello a la vista del Art. 10 de la LCS. Pues bien, el análisis de esta última jurisprudencia nos indica que es útil hacerse 5 preguntas clave para diagnosticar el resultado previsible de cualquier pleito sobre los cuestionarios de salud en los seguros de vida. Apliquemos las 5 preguntas a la situación pandémica global del COVID-19:

a) ¿El asegurador ha presentado un auténtico cuestionario que contenga preguntas sobre las circunstancias de salud del tomador? En situaciones de normalidad, el TS viene rechazando la validez de algunas declaraciones predispuestas y genéricas del asegurado sobre sus circunstancias de salud como auténticos cuestionarios. Ello no impide la aplicación, en algunos de estos casos, de cláusulas de exclusión de enfermedades preexistentes, siempre y cuando aparezcan reflejadas con las garantías propias de las cláusulas limitativas de los derechos de los asegurados conforme al Art. 3 de la LCS. A partir de ahora y a raíz de la situación pandémica global del COVID-19, cuando sabemos a ciencia cierta que hay miles - cuando no millones- de individuos no infectados y de infectados asintomáticos, no parece que vayan a seguir siendo válidas, en determinados casos, las declaraciones genéricas del asegurado afirmando que goza de buena salud.

b) ¿Las preguntas contenidas en el cuestionario son razonablemente detalladas? En situaciones de normalidad, nue4stro TS ha se considerado suficiente que las pregun-

\footnotetext{
13 Hemos venido exponiendo este método en nuestro blog (ajtapia.com) en reiteradas ocasiones. Así, el lector interesado puede ver, entre otras muchas, las siguientes entradas: de 07.04.2016 sobre "Las 5 preguntas clave para hacer un diagnóstico precoz de los pleitos sobre los cuestionarios de salud en los seguros de vida a la vista de la Jurisprudencia del Tribunal Supremo"; de 26.09.2016 sobre "Cuestionarios de salud en los seguros de vida: jurisprudencia reciente y diagnóstico diferencial"; de 26.01.2017 sobre "Cuestionarios y declaraciones de salud válidos en los seguros de vida. Sentencia de Tribunal Supremo núm. 726/2016, de 12 de diciembre"; etc.
} 
tas se refieran a las patologías más comunes, sin necesidad de un desglose detallado de enfermedades y sus circunstancias que llevaría a tener que utilizar cuestionarios integrados por cientos de preguntas minuciosas sobre aquellas patologías; lo que redundaría, en última instancia, en un sistema ineficiente y perjudicial para asegurados y aseguradores. A partir de ahora y a raíz de la situación pandémica global del COVID-19, nos parece recomendable que los aseguradores incluyan en los cuestionarios de salud preguntas expresas y específicas sobre el virus.

c) ¿El cuestionario presentado ha sido verdaderamente cumplimentado bien por el tomador o bien con la información que este ha proporcionado al asegurador o al mediador? En situaciones de normalidad, nuestro TS ha venido admitiendo ambas hipótesis, esto es, que las respuestas sean manuscritas por el tomador o bien que sean cumplimentadas por el mediador - de forma manuscrita o impresa- con la información facilitada por el tomador. A partir de ahora y a raíz de la situación de confinamiento de la población por la pandemia global del COVID-19, nos parece que los criterios de cumplimentación deberán adaptarse al uso de las tecnologías digitales propias del Insurtech de firma a distancia.

d) ¿Cómo ha omitido el tomador una enfermedad o lesión preexistente? En situaciones de normalidad, nuestro TS ha establecido que el grado de culpabilidad de la conducta omisiva del tomador es esencial para reducir proporcionalmente su prestación, si ha actuado con negligencia o culpa leve; o para denegar la prestación y liberar al asegurador, si el tomador ha actuado con dolo o culpa grave. A partir de ahora y a raíz de la situación pandémica global del COVID-19, nos parece que el dolo o la culpa grave del asegurado exigirá acreditar que ocultó conscientemente la infección vírica que se le hubiera diagnosticado.

e) ¿La enfermedad o lesión preexistente omitida ha causado el fallecimiento o la invalidez que constituyen el siniestro? En situaciones de normalidad, nuestro TS ha exigido que exista una relación de causalidad eficiente entre ambos factores para que la omisión resulte relevante. A partir de ahora y a raíz de la situación pandémica global del COVID-19, se plantea el delicadísimo problema médico, lógico y jurídico de la depuración de la incidencia causal del COVID-19 en el eventual fallecimiento de pacientes con cuadros de enfermedades -leves o graves $i ?-$ preexistentes. Para lo cual, en los pleitos futuros que con seguridad se derivaran de la pandemia, jugará un papel esencial la prueba pericial médica.

A lo anterior es preciso añadir una disposición legal relevante que plantea un problema de enorme relevancia social, que consiste en evitar que la eventual infección por el COVID-19 (incierta por la escasez de pruebas antes señalada) se transforme en una circunstancia discriminatoria cuando la persona -infectada o no $i$ ? - necesite contratar u seguro de personas, sea de vida, accidentes, enfermedad y asistencia sanitaria, dependencia o decesos ${ }^{14}$. En este punto, hay que traer a colación lo que establece la Disposición adicional quinta de la Ley de Contrato de Seguro ${ }^{15}$ al referirse a la "No

\footnotetext{
14 V. la entrada de nuestro blog (ajtapia.com) de 22.04.2020 sobre "No discriminación por el COVID-19 en los seguros de personas".

15 En texto añadido por la disposición final 1 de la Ley $\mathrm{N}^{\circ}$ 4/2018, de 11 de junio (BOE-A-2018-7832) publicado el 12/06/201 y en vigor a partir del 12/06/2018.
} 
discriminación por razón de VIH/SIDA u otras condiciones de salud"16. Si tomamos en consideración que una interpretación racional y sistemática de la disposición transcrita nos señala que las "otras condiciones de salud" han de ser análogas al VIH/ SIDA y si añadimos a lo anterior que científicos reputados -incluidos el ganador de un premio Nobel- establecen analogías evidentes entre el VIH y el COVID-19; la conclusión es manifiesta.

\section{B) La póliza de protección de los sanitarios que luchan con- tra el COVID-19. Una iniciativa admirable de las asegurado- ras españolas a través de UNESPA}

\section{Un colectivo admirable e incluso heroico: los profesiona- les sanitarios en lucha frente al COVID-19}

Podemos afirmar, sin temor a equivocarnos, que existe un sentimiento unánime en la sociedad española sobre la conducta admirable -e incluso heroica, a la vista de la penuria de los medios de autoprotección que han sufrido y siguen sufriendo- que han desarrollado los profesionales sanitarios en la lucha del COVID-19. Lamentablemente, a 15 de mayo se contaba un total de 50.455 sanitarios infectados, lo que representa un $21,4 \%$ del número total de infectados (cifra anormalmente elevada si la comparamos con Italia, que no pasa del 10\% o los EE UU, que no supera el 3\%). Por lo tanto, toda medida de cobertura de los riesgos que genera la lucha contra el COVID-19 a los profesionales sanitarios implicados en la misma parece justa y necesaria ${ }^{17}$.

\section{Una póliza de seguro de vida admirable}

Por lo anterior, nos parece admirable la iniciativa de 109 aseguradoras españolas a través de UNESPA de suscribir tres Pólizas de seguro de vida en régimen de coaseguro, conforme al Art. $33 \mathrm{LCS}^{18}$, que han creado para proteger a los profesionales sanitarios

\footnotetext{
${ }^{16}$ Que dice: "No se podrá discriminar a las personas que tengan VIH/SIDA u otras condiciones de salud. En particular, se prohíbe la denegación de acceso a la contratación, el establecimiento de procedimientos de contratación diferentes de los habitualmente utilizados por el asegurador o la imposición de condiciones más onerosas, por razón de tener VIH/SIDA u otras condiciones de salud, salvo que se encuentren fundadas en causas justificadas, proporcionadas y razonables, que se hallen documentadas previa y objetivamente". 17 V. la entrada de nuestro blog (ajtapia.com) de 20.05.2020 sobre "La póliza de protección de los sanitarios que luchan contra el COVID-19. Una iniciativa admirable de las aseguradoras españolas a través de UNESPA".

18 En las que actúan como entidades abridoras GENERALI ESPAÑA S.A. DE SEGUROS Y REASEGUROS, MAPFRE VIDA, S.A. de Seguros y Reaseguros sobre la Vida Humana y VIDACAIXA S.A.U. DE SEGUROS Y REASEGUROS. Sobre el Art. 33 LCS, v. Fernando Sánchez Calero en su comentario a dicho precepto [Ley de Contrato de Seguro. Comentarios a la Ley $N^{\circ}$ 50/1980, de 8 de octubre y a sus modificaciones, Sánchez Calero, F. (Dir.), Aranzadi, $4^{a}$ ed., 2010, pp. 521 y ss.].
} 
y de las residencias de mayores que luchan más directamente contra el COVID-19 en España. Para ello, las aseguradoras han constituido un fondo solidario dotado de 38 millones de euros que sirve para el pago de las primas del seguro.

Las aportaciones de las aseguradoras han permitido a UNESPA suscribir-en nombre y representación de 109 entidades- un seguro de vida gratuito que cubrirá dos tipos de contingencias: Por un lado, el fallecimiento de los sanitarios, con un capital asegurado en caso de fallecimiento por causa directa del COVID-19 de 30.000 euros por persona, que percibirán sus beneficiarios; y, por otro lado, un subsidio de hospitalización, que conllevará el pago de 100 euros al día al profesional ingresado siempre que su estancia hospitalaria supere los tres días y hasta un máximo de dos semanas. Es decir, los pagos oscilarán entre los 400 y los 1.400 euros por persona.

El ámbito temporal de la Póliza cubrirá a todas aquellas personas del colectivo asegurado que sean hospitalizadas o fallezcan a causa directa del coronavirus desde el pasado 14 de marzo hasta el próximo 13 de septiembre de 2020, ambos días incluidos. La cobertura tiene, por lo tanto, carácter retroactivo.

Se estima que el seguro de vida y el subsidio por hospitalización darán cobertura a través de esta Póliza a más de 700.000 personas en todo el país. Se trata, por lo tanto, de la mayor póliza colectiva -conforme al Art. 81 LCS $^{19}$ - de este tipo jamás suscrita en la historia de España para respaldar a quienes se encuentran en la primera línea de la lucha contra el COVID-19.

\section{Una póliza de arquitectura original}

En primer lugar, decimos que nos parece una iniciativa admirable desde el punto de vista técnico jurídico asegurador porque implica soluciones por completo originales, entre las que nos parece oportuno destacar las siguientes:

\subsection{Elementos personales de la Póliza}

\section{a) La tomadora UNESPA}

Actúa como tomadora UNESPA en representación de las 109 entidades aseguradoras que han hecho sus respectivas portaciones a un fondo solidario dotado de 38 millones de euros que sirve para el pago de las primas del seguro. Recordamos que UNESPA es la Asociación Empresarial del Seguro que representa a aseguradoras y reaseguradoras que reúnen aproximadamente el 98\% del volumen de negocio asegurador en el mercado español. Interesa recordar que el Art. 7 de la Ley $N^{\circ}$ 50/1980, de 8 de octubre,

\footnotetext{
19 Fernando Sánchez CAlero en su comentario a dicho precepto [Ley de Contrato de Seguro. Comentarios a la Ley $N^{\circ} 50 / 1980$, de 8 de octubre y a sus modificaciones, Sánchez Calero, F. (Dir.), Aranzadi, $4^{\mathrm{a}}$ ed., 2010, pp. 1558 y ss.].
} 
de Contrato de Seguro (LCS) dispone que "el tomador del seguro puede contratar el seguro por cuenta propia o ajena"20.

\section{b) Los profesionales sanitarios asegurados}

Se trata de un seguro de personas colectivo contratado conforme a lo previsto en el Art. 81 de la LCS que dice: "El contrato puede celebrarse con referencia a riesgos relativos a una persona o a un grupo de ellas. Este grupo deberá estar delimitado por alguna característica común extraña al propósito de asegurarse". En particular, conviene destacar que la Póliza tiene una cobertura personal amplísima ya que forman parte del grupo asegurado los dos grandes colectivos de profesionales sanitarios siguientes (conforme al Anexo de la Nota de prensa publicada el 18 de mayo por UNESPA):

\section{b.1) Colectivo asegurado en centros sanitarios}

Forman parte del colectivo asegurado los profesionales de las categorías que después se detallan que, durante el periodo de cobertura de la póliza, hayan prestado sus servicios en territorio español en: Centros hospitalarios públicos, hospitales de campaña dependientes de hospitales o de las comunidades autónomas, centros de salud y consultorios de atención primaria públicos, servicios públicos de urgencias y emergencias (112/061) y hospitales y clínicas privadas, así como los servicios de urgencias y emergencias dependientes de estos.

\section{b.2) Colectivo asegurado en residencias de mayores}

Forman parte del colectivo asegurado los profesionales que aparecen listados más abajo y que durante el periodo de cobertura de la póliza hayan prestado sus servicios en territorio español en residencias de mayores públicas o privadas, siempre que en el ejercicio de su actividad laboral, estatutaria o profesional hayan estado involucrados directamente en la lucha contra el coronavirus. Es decir, que hayan mantenido contacto físico con personas infectadas por el COVID-19.

\subsection{Contenido de la Póliza}

Como sucede en todo contrato de seguro, en la Póliza

"el asegurador se obliga, mediante el cobro de una prima y para el caso de que se produzca el evento cuyo riesgo es objeto de cobertura a indemnizar, dentro de los límites pactados, el daño producido al asegurado o a satisfacer un capital, una renta $u$ otras prestaciones convenidas" (Art. 1 de la LCS).

\footnotetext{
20 Sobre el Art. 7 LCS, ver Fernando SÁnChEZ CALERo en su comentario a dicho precepto [Ley de Contrato de Seguro. Comentarios a la Ley $N^{\circ}$ 50/1980, de 8 de octubre y a sus modificaciones, SÁnCHEZ CALERo, F. (Dir.), Aranzadi, $4^{\mathrm{a}}$ ed., 2010, pp. 162 y ss.] contempla la posible contratación del seguro por persona física o jurídica que actúe como representante del tomador, así como la eventual pluralidad de asegurados propia de las pólizas colectivas y la distribución de derechos y obligaciones.
} 
Veamos, pues, las dos obligaciones recíprocas de cada parte:

\section{a) Prestaciones cubiertas}

Son dos: a.1) la prestación principal por fallecimiento, en virtud de la cual la entidad aseguradora se obliga a pagar al beneficiario un capital bruto de 30.000 euros si, durante el periodo de cobertura, se produce el fallecimiento del asegurado por causa directa del COVID-19 contraído por el asegurado por motivo del desarrollo de su actividad laboral, estatutaria o profesional, habiendo estado involucrado directamente en la lucha contra el COVID-19 y manteniendo contacto físico con personas infectadas por el COVID-19. a.2) Prestación complementaria por hospitalización del asegurado en instalaciones hospitalarias públicas o privadas por la que la entidad aseguradora se obliga a pagar al beneficiario un importe bruto de 100 euros por día de hospitalización del asegurado, siempre que dicha hospitalización se haya iniciado durante el periodo de cobertura y se haya producido por causa directa del COVID-19 contraído por el asegurado por motivo del desarrollo de su actividad laboral, estatutaria o profesional al haber estado él mismo involucrado directamente en la lucha contra el COVID-19 y manteniendo contacto físico con personas infectadas por el COVID-19. Esta prestación se abonará siempre que la hospitalización requerida haya sido superior a tres días y el importe máximo a indemnizar corresponderá a 14 días de hospitalización. En el concepto de instalaciones hospitalarias públicas o privadas se incluyen instalaciones sanitarias temporales o portátiles que han sido habilitadas como establecimientos hospitalarios adicionales con ocasión de la pandemia (hospitales de campaña dependientes de hospitales o de las CCAA) pero no se entienden incluidos los hoteles medicalizados, ni tampoco los domicilios particulares, aunque se realicen hospitalizaciones domiciliarias.

Se establece expresamente que los riesgos de fallecimiento y hospitalización no serán excluyentes entre sí. De tal manera que, en el caso de que se produjera el fallecimiento antes de que se hubiera satisfecho la prestación de hospitalización que, en su caso, correspondiera, el importe de la prestación de hospitalización incrementará la prestación de fallecimiento a satisfacer a los beneficiarios de fallecimiento.

Un rasgo por completo original de esta Póliza -que muestra su vocación omnicomprensiva- es que, en ella y dada la naturaleza de las coberturas aseguradas en esa póliza, no se aplicará a las mismas ninguna exclusión.

\section{b) Prima}

La Póliza opera sobre la base de una prima inicial que paga el tomador del seguro, que recordemos es UNESPA actuando en nombre y representación de las 109 entidades aseguradoras. Otro rasgo por completo original de la Póliza consiste en que, en caso de que la siniestralidad sea menor que el fondo constituido, el importe sobrante será devuelto al Tomador a través de UNESPA, que lo destinará a fines sociales relacionados con la lucha contra el COVID-19. 


\section{Una demostración de responsabilidad social corporativa}

En segundo lugar, decimos que es admirable la Póliza como muestra de responsabilidad social corporativa que se manifiesta en las siguientes características:

a) Es una Póliza singular porque cubre un riesgo singular, como son las consecuencias en forma de fallecimiento o de hospitalización de la pandemia del COVID-19 en la salud del personal sanitario que ha estado combatiéndolo de manera directa; en una forma singular, puesto que se trata de una Póliza en que el pago de la prima no corre -ni directa ni indirectamente- a cargo del grupo asegurado, sino que se financia mediante un fondo constituido por aportaciones de 109 entidades aseguradoras que representan la práctica totalidad de las entidades integradas en UNESPA.

b) Es una Póliza estratégica en la lucha contra la pandemia del COVID-19 porque, si partimos del hecho cierto de que el Grupo asegurado lo integra personal sanitario que, en los diferentes centros señalados en la Póliza y desde sus diferentes categorías, combaten -directamente- la extensión de la pandemia del COVID entre la población en general; y añadimos que dicho colectivo asegurado ocupa una posición neurálgica en la vanguardia de la lucha contra el COVID-19; es un hecho notorio y evidente que existe un coeficiente multiplicador directo -con implicaciones a los efectos del cálculo del riesgo epidemiológico y la estadística de la siniestralidad aseguradora- entre el personal sanitario que se infecta y, por ende, debe dejar de prestar sus servicios porque enferma o fallece y la población que se infecta en origen, enferma por la infección o fallece a causa de ella.

c) Es una Póliza omnicomprensiva desde varios puntos de vista porque: en primer lugar, es una Póliza omnicomprensiva desde el punto de vista subjetivo tanto en lo que se refiere al Grupo Asegurable como al Grupo Asegurado ya que abarca todas las categorías de personal sanitario implicado en la lucha directa contra el COVID-19 presente en todas las instalaciones en las que dicho combate se produce. En segundo lugar, es una Póliza omnicomprensiva desde el punto de vista objetivo en el momento de incluir -en el "objeto del seguro"- la prestación principal de fallecimiento o complementaria de hospitalización para el personal sanitario que hayan prestado sus servicios en territorio español en centros hospitalarios públicos, en centros de salud y consultorios de atención primaria públicos o en los servicios públicos de urgencias y emergencias 112/061 del Sistema Nacional de Salud, o en hospitales privados o clínicas privadas o servicios de urgencias y emergencias dependientes de éstos, (o en residencias de mayores públicas o privadas) siempre que en el ejercicio de su actividad laboral, estatutaria o profesional para las anteriores instituciones sanitarias hayan estado involucrados directamente en la lucha contra el coronavirus/COVID-19, habiendo mantenido contacto físico con personas infectadas por el COVID-19. En tercer y último lugar, esta vocación omnicomprensiva de la Póliza se observa también en la compatibilidad de la prestación principal de fallecimiento y la complementaria de hospitalización y la inexistencia de causas de exclusión de la cobertura. 
Esta Póliza es también una muestra de la sostenibilidad aseguradora porque incorpora uno de los dos objetivos que definen la sostenibilidad financiera que es -junto al medioambiental- el objetivo social ${ }^{21}$.

\section{Conclusión: una Póliza de Seguro de Vida histórica}

Podemos concluir diciendo - sin miedo a caer en el abuso habitual del calificativoque estamos ante una Póliza de Seguro de Vida histórica por sus dimensiones, al ser la mayor póliza colectiva de este tipo jamás suscrita en la historia de España; por su finalidad, que consiste en respaldar a los profesionales sanitarios que se encuentran en la primera línea de la lucha contra el COVID-19; y por sus características técnico-jurídicas, ya que articula soluciones originales para alcanzar tan loable propósito.

\section{MEDIDAS EXTRAORDINARIAS EN LOS PLANES DE PENSIONES}

\section{A) El marco inicial de la disponibilidad de los planes de pensiones por la crisis del COVID-19 establecido en el RDL $11 / 2020$}

La Disposición adicional vigésima del RDL 11/2020 reguló la “disponibilidad de los planes de pensiones en caso de desempleo o cese de actividad derivados de la situación de crisis sanitaria ocasionada por el COVID-19" permitiendo que, durante el plazo de seis meses desde la entrada en vigor del Real Decreto 463/2020, de 14 de marzo, los partícipes de los planes de pensiones excepcionalmente, hagan efectivos sus derechos consolidados fijando dos extremos de aquella disponibilidad anticipada:

a) Primero, los supuestos consistentes en que el partícipe se encuentre en situación legal de desempleo como consecuencia de un expediente de regulación temporal de empleo derivado de la situación de crisis sanitaria ocasionada por el COVID-19; o que sea empresario titular de establecimientos cuya apertura al público se haya visto suspendida como consecuencia de lo establecido en el Art. 10 del Real Decreto 463/2020, de 14 de marzo; o, en el caso de los trabajadores por cuenta propia, que hubieran estado previamente integrados en un régimen de la Seguridad Social como tales y hayan cesado en su actividad como consecuencia de la situación de crisis sanitaria ocasionada por el COVID-19.

\footnotetext{
${ }_{21}$ Recordemos que, en la entrada de nuestro blog del pasado 8 de abril de 2020 sobre "La sostenibilidad financiera en el mundo posterior a la pandemia del coronavirus", decíamos: "Dando un paso más en nuestro razonamiento, descubrimos que, para ser sostenibles las finanzas, los agentes económicos deben añadir a los tres factores clásicos de evaluación de la calidad de una inversión que determinan en última instancia la decisión de realizarla (rentabilidad, riesgo y liquidez) dos objetivos más de sostenibilidad que son el medioambiental y el social".
} 
b) Segundo, el importe de los derechos consolidados disponible que no podrá ser superior a los límites legalmente establecidos según las diferentes situaciones (salarios dejados de percibir mientras se mantenga la vigencia del expediente de regulación temporal de empleo, etc.).

Se aclaraba que este régimen de disponibilidad anticipada excepcional de los derechos consolidados de los planes de pensiones resultará aplicable los asegurados de los planes de previsión asegurados, planes de previsión social empresarial y mutualidades de previsión social a que se refiere el Art. 51 de la Ley $\mathrm{N}^{\circ} 35 / 2006$, de 28 de noviembre, del Impuesto sobre la Renta de las Personas Físicas ${ }^{22}$.

\section{B) El desarrollo de las normas sobre la disponibilidad de los planes de pensiones por la crisis del COVID-19 por el RDL $15 / 2020$}

Después, el Art. 23 del RDL 15/2020 desarrolló los siguientes aspectos de las “normas sobre disponibilidad excepcional de los planes de pensiones en situaciones derivadas de la crisis sanitaria ocasionada por el COVID-19":

a) La legitimación activa: Dispone el RDL 15/2020 que podrán solicitar hacer efectivos sus derechos consolidados en los supuestos previstos por el RDL 11/2020: Los partícipes de los planes de pensiones del sistema individual y asociado y los partícipes de los planes de pensiones del sistema de empleo. Este segundo colectivo puede clasificarse en los dos grupos siguientes: Los partícipes de los planes de pensiones del sistema de empleo de aportación definida o mixtos para aquellas contingencias definidas en régimen de aportación definida. Los partícipes de los planes de pensiones del sistema de empleo de la modalidad de prestación definida o mixtos para aquellas contingencias definidas en régimen de prestación definida o vinculadas a la misma, "en caso de estar afectados por un ERTE, la suspensión de apertura al público de establecimientos o el cese de actividad, derivados de la situación de crisis sanitaria ocasionada por el COVID-19, cuando lo permita el compromiso por pensiones y lo prevean las especificaciones del plan aprobadas por su comisión de control en las condiciones que estas establezcan".

b) La acreditación de las circunstancias habilitantes: Establece el RDL 15/2020 que el partícipe del plan de pensiones que solicite la disposición anticipada de sus derechos consolidados a resultas de la crisis del COVID-19 deberá presentar ante la entidad gestora del fondo de pensiones en el que el plan respectivo esté integrado una serie de documentos que se adaptan a cada una de las situaciones cubiertas: Supuestos en los que el partícipe resulte afectado por un expediente de regulación temporal de empleo (ERTE) derivado de la situación de crisis sanitaria ocasionada por el COVID-19. Supuestos en los que el partícipe empresario sea titular de un establecimiento cuya apertura al público se haya visto suspendida como consecuencia de lo establecido en el Art. 10 del Real Decreto 463/2020. Supuestos en los que el trabajador por cuenta

22 El lector puede ver, sobre los planes de previsión asegurados y los planes de previsión social empresarial nuestra "Guía del Contrato de Seguro", Ed. Thomson Reuters Aranzadi, 2018, pp. 204 y ss. 
propia que hubiera estado previamente integrado en un régimen de la Seguridad Social como tal, o en un régimen de mutualismo alternativo a esta; haya cesado en su actividad durante el estado de alarma decretado por el Gobierno por el COVID-19.

c) Como cláusula de cierre lógico del sistema de disponibilidad anticipada de los derechos consolidados y de protección al partícipe, se establece que, en el caso de que el partícipe solicitante no pudiese aportar alguno de los documentos requeridos, podrá sustituirlo mediante una declaración responsable que incluya la justificación expresa de los motivos, relacionados con las consecuencias de la crisis del COVID-19, que le impiden tal aportación.

d) A efectos de control preventivo se añade que "el partícipe será responsable de la veracidad de la documentación acreditativa de la concurrencia del supuesto de hecho que se requiera para solicitar la prestación, así como de la exactitud en la cuantificación del importe a percibir".

e) El importe disponible de los derechos consolidados: El RDL 15/2020 establece que dicho importe será el justificado por el partícipe a la entidad gestora de fondos de pensiones, con el límite máximo de la menor de dos cuantías para el conjunto de planes de pensiones de los que sea titular.

f) El plazo de reembolso de los derechos consolidados: El reembolso deberá efectuarse dentro del plazo máximo de siete días hábiles desde que el partícipe presente la documentación acreditativa completa; plazo que se ampliará hasta treinta días hábiles, en el caso de los planes de pensiones de la modalidad de empleo ${ }^{23}$.

\section{C) Reformas de la disponibilidad de planes de pensiones por el RDL 16/2020}

La nueva regulación establece la disponibilidad de sus derechos consolidados en los planes de pensiones por el trabajador por cuenta propia que hubiera estado previamente integrado en un régimen de la Seguridad Social como tal, o en un régimen de mutualismo alternativo a esta. Así, la disposición final quinta del RDL 16/2020 comienza con un título amenazante de la lógica jurídico-temporal y de una mínima seguridad jurídica (tan necesaria, por otra parte, en los tiempos que corren): “Modificación del Real Decreto-Ley $N^{\circ} 15 / 2020$, de 21 de abril, de medidas urgentes complementarias para apoyar la economía y el empleo".

\section{CONCLUSIONES}

$1^{\mathrm{a}}$. España ha sido, por desgracia, uno de los países pioneros en sufrir la pandemia del COVID-19 y esta dramática experiencia puede servir para sacar, en el mundo del seguro, enseñanzas que puedan resultar útiles para los países hermanos de Iberoamérica.

\footnotetext{
${ }^{23}$ El lector puede ver, sobre la regulación de la disponibilidad de los derechos consolidados de los planes de pensiones nuestro “Manual de Derecho de Seguros y Fondos de Pensiones”, Ed. Iustel, 2014, pp. 250 y ss.
} 
$2^{\mathrm{a}}$. Entre las medidas extraordinarias que inciden en el estatuto de las entidades aseguradoras destaca, en primer lugar, el principio de prudencia en la remuneración de los administradores y del personal de las entidades aseguradoras en tiempo del coronavirus establecido en las directrices europeas emitidas por la Autoridad Europea de Seguros y Pensiones de Jubilación (EIOPA).

$3^{\text {a }}$. Entre las medidas extraordinarias que inciden en el estatuto de las entidades aseguradoras destaca, en segundo lugar, el principio de flexibilidad en la exigencia del cumplimiento de las obligaciones de información por las personas y entidades sujetas a la supervisión de la Dirección General de Seguros y Fondos de Pensiones que se establecen en las normas españolas.

$4^{\mathrm{a}}$. Entre las medidas extraordinarias que inciden en los seguros de daños destaca, en primer lugar, la Comunicación de la Comisión Europea de 28 de marzo de 2020 para adaptar el seguro de crédito a la exportación a corto plazo a las circunstancias extraordinarias provocadas por el brote de COVID-19.

$5^{\text {a }}$. Entre las medidas extraordinarias que inciden en los seguros de daños destaca, en segundo lugar, el refuerzo en España del Seguro de Crédito a la Exportación como medida extraordinaria de apoyo público a la financiación empresarial ante la crisis del COVID-19 por el Real Decreto-Ley No ${ }^{\circ} / 2020$.

6 . Entre las medidas extraordinarias que inciden en los seguros de personas y, especialmente, de vida, destaca, en primer lugar, la aplicación del método de diagnóstico precoz de las 5 preguntas a la situación pandémica global del COVID-19.

$7^{\mathrm{a}}$. Como experiencia práctica extraordinaria en España en el ámbito de los seguros de personas resalta la Póliza de protección de los sanitarios que luchan contra el COVID-19.

$8^{\mathrm{a}}$. Entre las medidas extraordinarias que inciden en los planes de pensiones, destacan el régimen de disponibilidad anticipada de los derechos consolidados y económicos por la crisis del COVID-19 establecido, dentro del Derecho español, por el RDL 11/2020, el RDL 15/2020 y el RDL 16/2020.

\section{BIBLIOGRAFÍA}

Sánchez Calero, F., Ley de Contrato de Seguro. Comentarios a la Ley $N^{\circ}$ 50/1980, de 8 de octubre y a sus modificaciones, Sánchez CALERo, F. (Dir.), Aranzadi, 4ª ed., 2010.

Tapia Hermida, A.J., Manual de Derecho de Seguros y Fondos de Pensiones, Ed. Iustel, 2014.

Id., Guía del Contrato de Seguro, Ed, Thomson Reuters AranZadi, Cizur Menor 2018. 
